\title{
Electrical transport properties of black phosphorus based field-effect transistor with $\mathrm{Au} / \mathrm{Co} / \mathrm{MgO}$ tunneling contacts
}

Cite as: J. Appl. Phys. 122, 164301 (2017); https://doi.org/10.1063/1.5000524

Submitted: 16 August 2017 . Accepted: 10 October 2017 . Published Online: 23 October 2017

Shiheng Liang, Huaiwen Yang, Abdelhak Djeffal, Bingshan Tao, Stefan Mc-Murtry, Stéphane Mangin (D), and Yuan Lu (iD)

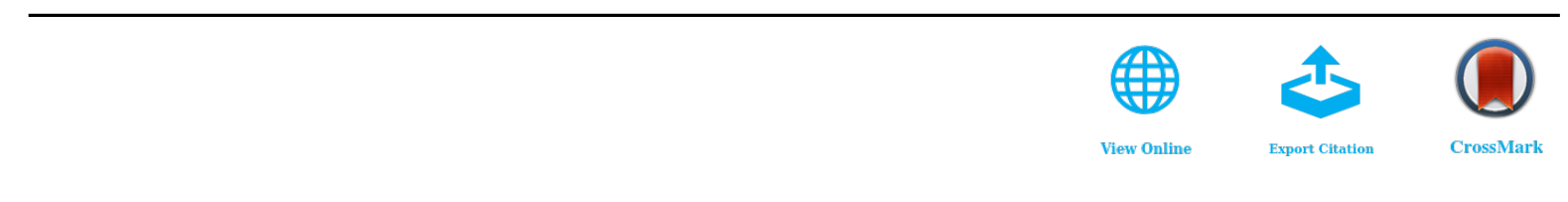

\section{ARTICLES YOU MAY BE INTERESTED IN}

Tunneling field effect transistor integrated with black phosphorus-MoS 2 junction and ion gel dielectric

Applied Physics Letters 110, 033103 (2017); https://doi.org/10.1063/1.4974303

Electric field effect in ultrathin black phosphorus

Applied Physics Letters 104, 103106 (2014); https://doi.org/10.1063/1.4868132

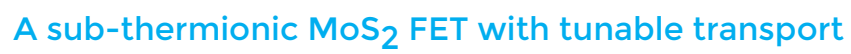

Applied Physics Letters 111, 163501 (2017); https://doi.org/10.1063/1.4996953

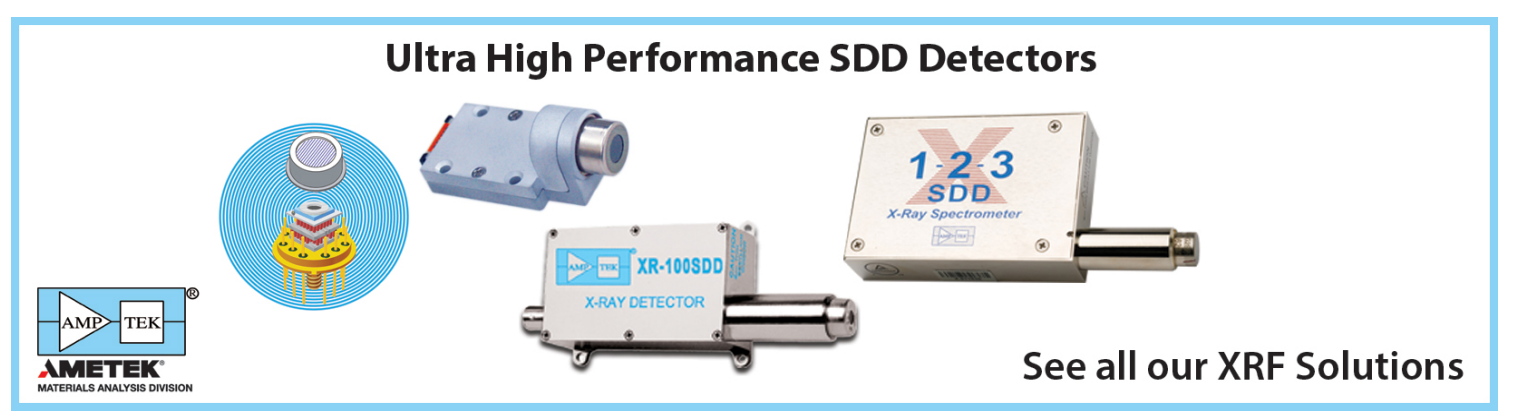

J. Appl. Phys. 122, 164301 (2017); https://doi.org/10.1063/1.5000524 


\title{
Electrical transport properties of black phosphorus based field-effect transistor with $\mathrm{Au} / \mathrm{Co} / \mathrm{MgO}$ tunneling contacts
}

\author{
Shiheng Liang, Huaiwen Yang, Abdelhak Djeffal, Bingshan Tao, Stefan Mc-Murtry, \\ Stéphane Mangin, and Yuan $\mathrm{Lu}^{\mathrm{a}}$ \\ Institut Jean Lamour, UMR 7198, CNRS-Université de Lorraine, BP 239, 54506 Vandouvre, France
}

(Received 16 August 2017; accepted 10 October 2017; published online 23 October 2017)

Black phosphorus (BP) has recently emerged as a promising two-dimensional direct bandgap semiconducting material. Here, we report the fabrication and the electrical transport measurements of the black phosphorus based field-effect transistor with the $\mathrm{Au} / \mathrm{Co} / \mathrm{MgO}$ as drain and source tunneling contacts. By modulating the back-gate voltage, the multilayer black phosphorus channel exhibits ambipolar characteristics (both $n$-type and $p$-type) and the conduction behavior can be switched from hole dominated to electron dominated transport region. In the hole dominated region, we have measured a minimum of Schottky barrier height of $37 \mathrm{meV}$ for $\mathrm{Au} / \mathrm{Co} / \mathrm{MgO}$ contact on $\mathrm{BP}$. Moreover, the transistor ON/OFF $\left(I_{\text {on }} / I_{\text {off }}\right)$ ratio is obtained as large as $10^{7}$ at $20 \mathrm{~K}$ and $10^{5}$ at $300 \mathrm{~K}$. A systematic study of the temperature and the back-gate voltage dependent conduction properties has been performed to understand the modulation of band structure and the ambipolar behavior. The demonstration of high ON/OFF ratio and low Schottky barrier height by using $\mathrm{Au} / \mathrm{Co} / \mathrm{MgO}$ tunneling contacts reveals a promising potential for spintronics applications with multilayer black phosphorus field-effect transistor. Published by AIP Publishing.

https://doi.org/10.1063/1.5000524

\section{INTRODUCTION}

Two-dimensional (2D) materials with atomic thickness have been recently unveiled as an important family of materials in physics and materials science. ${ }^{1-9}$ Since the discovery of graphene in $2004,{ }^{1}$ a fair amount of research has been dedicated to fabricate other thin layer 2D materials, for examples of boron nitride ${ }^{10}$ and transition metal dichalcogenides (TMDs) materials. ${ }^{3-9}$ The understanding of physics and material characteristics of $2 \mathrm{D}$ materials leads to the possibility of many applications, such as transistors, ${ }^{7,9}$ optoelectronics, ${ }^{11}$ sensors, ${ }^{12}$ photovoltaics, ${ }^{13}$ medicine, ${ }^{14}$ and energy storage. ${ }^{15}$ In the 2D material family, graphene is known as a semiconductor with zero bandgap. Despite its high mobility, the graphene is not suitable to be used as field-effect transistors (FETs $)^{1,2}$ due to the zero bandgap. Another 2D material is boron nitride, which is an insulator and can be used as a tunnel barrier in 2D-heterostructure device to improve the physical properties. ${ }^{10}$ Besides the above two types of $2 \mathrm{D}$ materials, TMDs such as $\mathrm{MoS}_{2}, \mathrm{WSe}_{2}$, and $\mathrm{MoSe}_{2}$ have semiconducting properties and can play the role of drain-source channel in FETs, but their mobility is relatively low. ${ }^{3-9}$

Recently, black phosphorus (BP) has attracted a large amount of attention and emerged as an important $2 \mathrm{D}$ material due to its interesting physics properties. ${ }^{16-24}$ The BP has a layer stacked structure by van der Waals interactions. The monolayer (ML) BP has a direct bandgap of about $2 \mathrm{eV}$. For multilayer BP, the band gap decreases with the increase of number of layers, changing from $2 \mathrm{eV}$ (ML) to about $0.3 \mathrm{eV}$ (bulk). This provides a wide range of tunability of bandgap for the multilayer BP. ${ }^{16}$ In addition, BP has a larger carrier

\footnotetext{
${ }^{a)}$ Email: yuan.lu@univ-lorraine.fr
}

mobility (highest values up to $\sim 1000 \mathrm{~cm}^{2} \mathrm{~V}^{-1} \mathrm{~s}^{-1}$ at room temperature $)^{18}$ compared to TMDs, which is better to serve as channel for FET applications. Moreover, by using BP as channel, the FET with ambipolar modulated behavior (both $n$-type and $p$-type) can be realized. ${ }^{17-21}$ Recently, spin transport experiments have also been demonstrated in ultrathin multilayer BP based non-local spin valve structures. ${ }^{22} \mathrm{~A}$ long spin diffusion length $(>2.5 \mu \mathrm{m})$ was measured owing to the high mobility and low spin-orbit coupling properties of BP. Therefore, great efforts have been carried out to push the BP based devices towards application in nano-electronics, ${ }^{17,19}$ optoelectronics, ${ }^{20,21}$ FETs,${ }^{18}$ and spintronics. ${ }^{22}$

In this work, we have fabricated the BP based FETs with $\mathrm{Au} / \mathrm{Co} / \mathrm{MgO}$ tunneling contacts and systematically studied the transport properties as a function of back-gate voltage and temperature. The ambipolar characteristics (both $n$-type and $p$-type) in multilayer BP has been observed with a high ON/OFF ratio as large as $10^{7}$. The Schottky barrier height is measured as low as $37 \mathrm{meV}$ for $\mathrm{Au} / \mathrm{Co} / \mathrm{MgO}$ contact on $\mathrm{BP}$. The high ON/OFF ratio and low Schottky barrier height demonstrate that BP has a promising potential for future FET and spintronics applications.

\section{EXPERIMENT}

Figure 1(a) shows the optical image of our BP based FET device. First, a flake of multilayer BP was mechanically exfoliated onto a $\mathrm{Si}\left(\mathrm{n}^{++}\right) / \mathrm{SiO}_{2}(280 \mathrm{~nm})$ substrate as the FET channel. The thickness of this flake was measured by an atomic force microscopy (AFM) to be about $7 \mathrm{~nm}$ [inset of Fig. 1(a)]. Considering $0.5 \mathrm{~nm}$ for one $\mathrm{ML} \mathrm{BP},{ }^{18}$ the thickness of the flake corresponds to about 14 MLs. Second, e-beam lithography (Raith-150) was performed to define five 
(a)
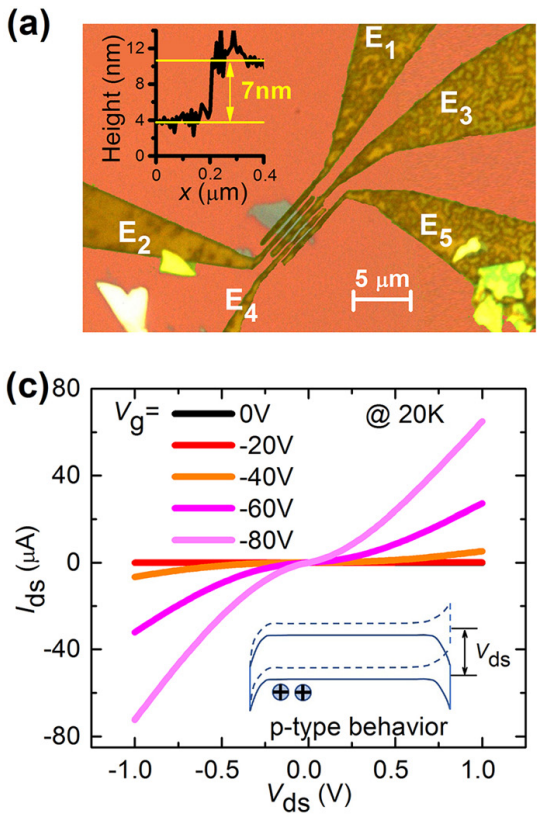

(b)

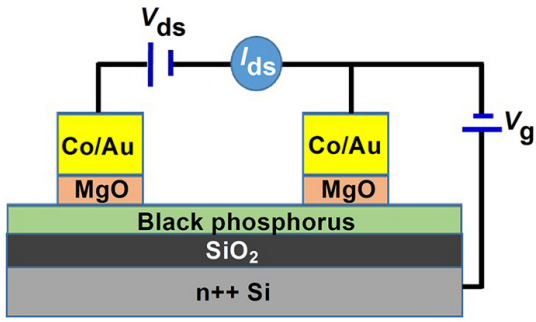

(d)

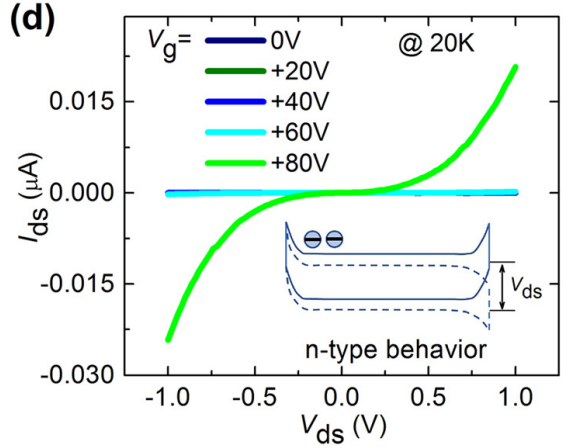

FIG. 1. (a) Optical image of black phosphorus flake exfoliated on $\operatorname{Si}\left(\mathrm{n}^{++}\right)$ $280 \mathrm{~nm} \mathrm{SiO}{ }_{2}$ substrate with contacts of $\mathrm{Au}(10 \mathrm{~nm}) / \mathrm{Co}(10 \mathrm{~nm}) / \mathrm{MgO}(2 \mathrm{~nm})$. Insert: the thickness of this BP flake was measured by AFM to be about $7 \mathrm{~nm}$. (b) Schematics of BP based field effect transistor. A drain-source bias $\left(V_{\mathrm{ds}}\right)$ was applied to inject the current $I_{\mathrm{ds}}$ through the BP channel. Meanwhile, a back-gate voltage $\left(V_{\mathrm{g}}\right)$ was applied between the substrate and one contact to modulate the carrier density in the BP channel. (c) Output characteristics of drain-source current-voltage $\left(I_{\mathrm{ds}}-V_{\mathrm{ds}}\right)$ measured with applying negative back-gate voltages $V_{\mathrm{g}}$. Insert: schematic of band profile in BP by applying $V_{\mathrm{ds}}$ under negative $V_{\mathrm{g}}$. (d) Output characteristics of drainsource current-voltage $\left(I_{\mathrm{ds}}-V_{\mathrm{ds}}\right)$ measured with applying positive back-gate voltages $V_{\mathrm{g}}$. Insert: schematic of band profile in BP by applying $V_{\mathrm{ds}}$ under positive $V_{\mathrm{g}}$. electrodes with a width of $500 \mathrm{~nm}$ on the selected flake. The distance between each electrode is about $450 \mathrm{~nm}$. Then the sample was introduced into a molecular beam epitaxy (MBE) system to deposit the ferromagnetic (FM) electrodes as drain and source tunneling contacts, which consists of $\mathrm{Au}(10 \mathrm{~nm}) / \mathrm{Co}(10 \mathrm{~nm}) / \mathrm{MgO}(2 \mathrm{~nm})$. The $2 \mathrm{~nm} \mathrm{MgO}$ is used as a tunnel barrier between metals and BP to effectively reduce the contact Schottky barrier height. ${ }^{25}$ After deposition and lift-off procedures, a second e-beam lithography was performed to define the large pads for electrical connection. $\mathrm{Au}(190 \mathrm{~nm}) / \mathrm{Ti}(10 \mathrm{~nm})$ was thermally evaporated in a PLASSYS MEB400s system for the large pads. Finally, the device was annealed at $120^{\circ} \mathrm{C}$ for one hour in vacuum and then followed by the coverage of $10 \mathrm{~nm} \mathrm{MgO}$ protection layer. Figure 1(b) shows the schematic of electric characterization of BP based transistor. A drain-source bias $\left(V_{\mathrm{ds}}\right)$ was applied between the two contacts to inject the current $I_{\mathrm{ds}}$ through the BP channel. Meanwhile, a back-gate voltage $\left(V_{\mathrm{g}}\right)$ was applied between the substrate and one electrode to modulate the carrier density in the BP channel.

\section{RESULTS AND DISCUSSIONS}

\section{A. Drain-source current-voltage $\left(I_{\mathrm{ds}}-V_{\mathrm{ds}}\right)$ characteristics}

The two-terminal drain-source current-voltage characteristics $\left(I_{\mathrm{ds}}-V_{\mathrm{ds}}\right)$ were studied at $20 \mathrm{~K}$ between the electrodes E1 and E2 with negative and positive back-gate voltages [Figs. 1(c) and 1(d), respectively]. From the measurement of $I_{\mathrm{ds}}-V_{\mathrm{ds}}$, the back-gate voltages show an efficient modulation on the $I_{\mathrm{ds}}$, which indicates the field-effect transistor behavior. At $V_{\mathrm{g}}=0 \mathrm{~V}$, the current density is rather low $\left(I_{\mathrm{ds}}<1 \mathrm{pA}\right.$ at $\left.V_{\mathrm{ds}}= \pm 1 \mathrm{~V}\right)$. As soon as we applied a negative or positive back-gate voltage $V_{\mathrm{g}}$, the $I_{\mathrm{ds}}-V_{\mathrm{ds}}$ characteristics dramatically change. The quasi-symmetric nonlinearity of $I_{\mathrm{ds}}-V_{\mathrm{ds}}$ is attributed to the back-to-back Schottky diode structures of the device, which is induced by the Schottky contacts of Co/ $\mathrm{MgO}$ on BP [inset of Figs. 1(c) and 1(d)]. It is found that applying negative $V_{\mathrm{g}}$ can get much higher current density than that with positive $V_{\mathrm{g}}$. Under a negative back-gate voltage of $V_{\mathrm{g}}=-80 \mathrm{~V}, I_{\mathrm{ds}}$ can reach $80 \mu \mathrm{A}$ at $V_{\mathrm{ds}}= \pm 1 \mathrm{~V}$, while there is about $0.02 \mu \mathrm{A}$ at $V_{\mathrm{ds}}= \pm 1 \mathrm{~V}$ under a positive backgate voltage of $V_{\mathrm{g}}=+80 \mathrm{~V}$. In fact, at different $V_{\mathrm{g}}$, the transport mechanism is different since $V_{\mathrm{g}}$ can effectively modulate the Fermi level $\left(E_{\mathrm{F}}\right)$ inside the bandgap of BP. Under the negative back-gate voltage, the $E_{\mathrm{F}}$ is attracted to be close to the energy of valence band maximum $\left(E_{\mathrm{V}}\right)$ of $\mathrm{BP}$, resulting in the hole conduction transport. However, under positive back-gate voltage, the $E_{\mathrm{F}}$ is pushed close to the energy of conduction band minimum $\left(E_{\mathrm{C}}\right)$ of $\mathrm{BP}$, resulting in the electron conduction transport. The larger conductivity for hole transport region indicates a higher carrier density in BP for hole transport, which means that the $E_{\mathrm{F}}$ in our BP covered with $\mathrm{MgO}$ should be much closer to $E_{\mathrm{V}}$ than $E_{\mathrm{C}}$ at zero $V_{\mathrm{g}}$.

\section{B. Field-effect transistor $\left(I_{\mathrm{ds}}-V_{\mathrm{g}}\right)$ characteristics}

The field-effect transistor characteristics were measured from the $I_{\mathrm{ds}} v s$. $V_{\mathrm{g}}$ curves at different temperature, as shown in Fig. 2(a). The asymmetric ambipolar behavior can be observed in all investigated temperature region. At large negative back-gate voltage $\left(V_{\mathrm{g}}=-80 \mathrm{~V}\right)$ when hole transport is dominated, the temperature dependence is not evident with $V_{\mathrm{ds}}=-1 \mathrm{~V}$. However, at a large positive back-gate voltage $\left(V_{\mathrm{g}}=+80 \mathrm{~V}\right)$ when electron transport is dominated, the conductance with $V_{\mathrm{ds}}=-1 \mathrm{~V}$ can be changed more than one order from $20 \mathrm{~K}$ to $300 \mathrm{~K}$. Since the variation of mobility with temperature is small with $V_{\mathrm{ds}}=-1 \mathrm{~V}$ (see below), the different temperature dependent conductance mainly reflects different carrier densities in the $p$-type and $n$-type transport region. If assuming that the carrier density in BP follows the thermal activation rule in the intrinsic semiconductor, the 

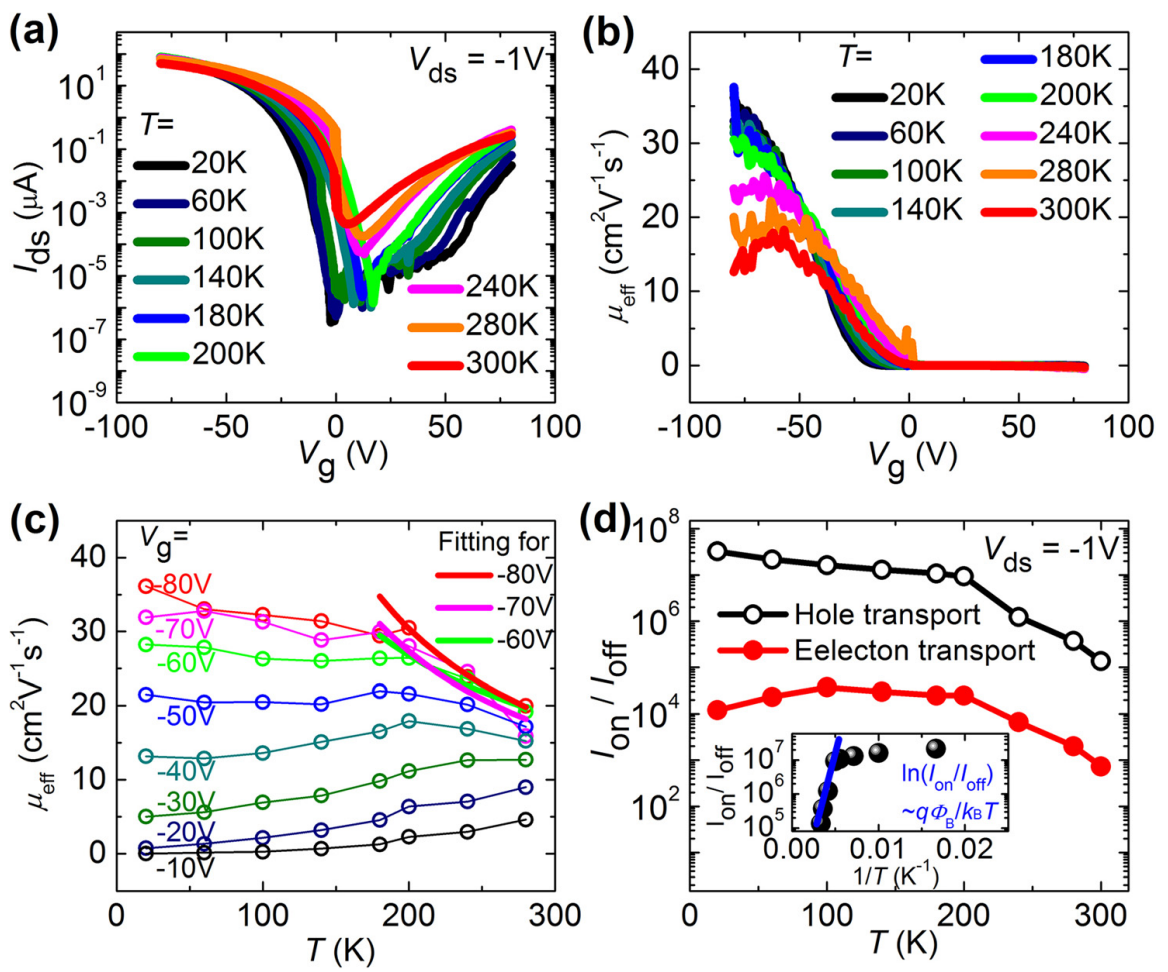

FIG. 2. (a) Transfer characteristics of $I_{\mathrm{ds}}-V_{\mathrm{g}}$ in logarithmic scale measured with $V_{\mathrm{ds}}=-1 \mathrm{~V}$ at different temperatures. (b) Extracted effective mobility $\mu_{\text {eff }}$ versus $V_{\mathrm{g}}$ at different temperatures with $V_{\mathrm{ds}}=-1 \mathrm{~V}$. (c) Temperature dependent mobility $\mu_{\text {eff }}$ measured under different back-gate voltages $V_{\mathrm{g}}$. (d) Temperature dependent $I_{\mathrm{on}} / I_{\text {off }}$ ratio measured for hole transport (black open) and electron transport (red solid) situations, respectively. Insert: $I_{\mathrm{on}} / I_{\mathrm{off}}$ ratio under hole transport in logarithmic scale plotted with $1 / T$. The blue line is a linear fitting for $\ln \left(I_{\text {on }} / I_{\text {off }}\right) v s .1 / T$. hole and electron densities can be expressed by $p=n_{i} e^{\frac{E_{i}-E_{F}}{k_{B} T}}$ and $n=n_{i} e^{\frac{E_{F}-E_{i}}{k_{B} T}}$, respectively. ${ }^{26} n_{i}=2.509 \times 10^{19}\left(\frac{m_{n} m_{p}}{m_{0}^{2}}\right)^{3 / 4}$ $\left(\frac{T}{300}\right)^{3 / 2} e^{-\frac{E_{g}}{2 k_{B} T}}\left(\mathrm{~cm}^{-3}\right)$ is the intrinsic carrier density, and $E_{i}$ is the intrinsic Fermi energy. $m_{n}, m_{p}$, and, $m_{0}$ are the electron mass in $E_{\mathrm{C}}$, the hole mass in $E_{\mathrm{V}}$, and the free electron mass, respectively. This can easily explain that the larger $\left|E_{i}-E_{F}\right|$ results in higher carrier density when increasing $V_{\mathrm{g}}$. In addition, the ratio of carrier density between $300 \mathrm{~K}$ and $20 \mathrm{~K}$ can be expressed as $p(300 K) / p(20 K)=\left(\frac{300}{20}\right)^{3 / 2} e^{\frac{E_{i}-E_{F}-0.5 E_{g}}{k_{B}}\left(\frac{1}{300}-\frac{1}{20}\right)}$ $\propto e^{\frac{0.5 E_{g}-\left(E_{i}-E_{F}\right)}{k_{B}}}$ for hole transport region and $n(300 K) / n(20 K)=\left(\frac{300}{20}\right)^{3 / 2} e^{\frac{E_{F}-E_{i}-0.5 E_{g}}{k_{B}}\left(\frac{1}{300}-\frac{1}{20}\right)} \propto e^{\frac{0.5 E_{g}-\left(E_{F}-E_{i}\right)}{k_{B}}}$ for electron transport region. This explains that the carrier density is more sensitive to the temperature variation when $\left|E_{i}-E_{F}\right|$ is smaller under positive $V_{\mathrm{g}}$ in the $n$-type transport region.

\section{Temperature dependent channel mobility}

The effective field-effect mobility can be extracted from the slope of $\mathrm{d} I_{\mathrm{ds}} / \mathrm{d} V_{\mathrm{g}}$ from the $I_{\mathrm{ds}}-V_{\mathrm{g}}$ curves, as shown in Fig. 2(b),

$$
\mu_{\mathrm{eff}}=\frac{d I_{d s}}{d V_{g}} \frac{L}{w C_{i} V_{d s}},
$$

where $L$ is the length of the channel $(450 \mathrm{~nm}), w$ is the width of the channel $(2.9 \mu \mathrm{m})$, and $C_{\mathrm{i}}$ is the gate capacitance $\left[1.3 \times 10^{-4} \mathrm{Fm}^{-2}\right.$ for $\mathrm{Si} / \mathrm{SiO}_{2}(280 \mathrm{~nm})$ substrate $]$. The effective field-effect mobility is found to increase under negative $V_{\mathrm{g}}$ [Fig. 2(c)]. At $20 \mathrm{~K}$, with $V_{\mathrm{ds}}=-1 \mathrm{~V}$ and $V_{\mathrm{g}}=-80 \mathrm{~V}$, we can obtain a mobility of $\mu_{\text {eff }} \sim 38 \mathrm{~cm}^{2} \mathrm{~V}^{-1} \mathrm{~s}^{-1}$. This value is lower than the previously reported values, which could be due to the scattering related to the charged impurities at $\mathrm{BP} / \mathrm{sub}$ strate interface at low temperature. ${ }^{22}$ The enhancement of mobility with the increase of $V_{\mathrm{g}}$ as well as the carrier density is due to the shielding effect of carrier to the Coulomb scattering from the charged impurities, ${ }^{26}$ which has also been observed in $\mathrm{MoS}_{2}$ based FET system. ${ }^{9}$ Figure 2(c) displays the variation of $\mu_{\text {eff }}$ with temperature measured under different $V_{\mathrm{g}}$. It is interesting to find that there exist two distinct temperature dependences. $\mu_{\text {eff }}$ decreases with the increasing $T$ when $V_{\mathrm{g}}<-50 \mathrm{~V}$, while it increases with $T$ when $V_{\mathrm{g}}>-50 \mathrm{~V}$. For $V_{\mathrm{g}}<-50 \mathrm{~V}, \mu_{\text {eff }}$ decreases faster when $T>200 \mathrm{~K}$, which is due to the carrier-phonon scattering at higher temperature. ${ }^{27}$ This can be expressed as $\mu \sim T^{-\alpha}$, the exponent $\alpha$ is fitted to be 1.27 and 1.21 , and 0.91 for the data at $V_{\mathrm{g}}=-60 \mathrm{~V},-70 \mathrm{~V}$ and $-80 \mathrm{~V}$, respectively. For atomically thin $2 \mathrm{D}$ materials, the exponent $\alpha$ is reported to be $\sim 1.69$ for $\mathrm{MoS}_{2},{ }^{27}$ and between 1 and 6 for graphene. ${ }^{28-30}$ Below $200 \mathrm{~K}$, the slower decrease of $\mu$ suggests an impurity-dominated scattering mechanism. ${ }^{18}$ For $V_{\mathrm{g}}>-50 \mathrm{~V}$ when $E_{\mathrm{F}}$ moves far away from $E_{\mathrm{V}}, \mu$ increases monotonically with the increase of $T$. This behavior means that when the carrier density is very low, the mobility is only limited by the scattering from the charged impurities. ${ }^{26}$

\section{Temperature dependent transistor ON/OFF ratio}

The transistor current ON/OFF ratio can be extracted from $I_{\text {on }}\left(\left|V_{\mathrm{g}}\right|=80 \mathrm{~V}\right) / I_{\text {off }}\left(V_{\mathrm{g}}=0 \mathrm{~V}\right)$ from the $I_{\mathrm{ds}}-V_{\mathrm{g}}$ curves. At $20 \mathrm{~K}$, a large ON/OFF ratio of $10^{7}$ is obtained for hole transport and a ratio of $10^{4}$ is measured for electron transport. The ON/OFF ratio for hole transport is found to be much larger than the recent reported values: $10^{3}$ with $\mathrm{Au} / \mathrm{BP}$ 
contacts, ${ }^{21} 10^{5}$ with $\mathrm{Au} / \mathrm{Pd} / \mathrm{Ti} / \mathrm{BP}$ contacts,${ }^{20}$ and $10^{6}$ with $\mathrm{Co} / \mathrm{TiO}_{2} / \mathrm{BP}$ contacts. ${ }^{24}$ This high $I_{\text {on }} / I_{\text {off }}$ ratio provides promising device characteristics for future applications. Figure 2(d) shows the temperature dependent $I_{\mathrm{on}} / I_{\text {off }}$ behavior. For the holes dominated FET, the $I_{\mathrm{on}} / I_{\text {off }}$ increases with the decrease of temperature. Since $I_{\text {on }}$ has little change with temperature, the improvement of $I_{\text {on }} / I_{\text {off }}$ at low temperature is mainly attributed to the reduction in $I_{\text {off }}$ current. When $T>200 \mathrm{~K}$, the $I_{\text {off }}$ current (at $V_{\mathrm{g}} \sim 0 \mathrm{~V}$ ) is mainly dominated by the thermionic injection through Schottky barrier, which is proportional to $\exp \left(-q \Phi_{\mathrm{B}} / \mathrm{k}_{\mathrm{B}} T\right),{ }^{31}$ where $k_{\mathrm{B}}$ is the Boltzmann constant and $\Phi_{\mathrm{B}}$ is Schottky barrier height [see also below for Eq. (2)]. Thus, the slope of $-q \Phi_{\mathrm{B}} / \mathrm{k}_{\mathrm{B}}$ can be extracted from a linear fitting of $\ln \left(I_{\mathrm{on}} / I_{\mathrm{off}}\right)$ versus $1 / T$, as shown in the insert of Fig. 2(d). The slope obtained from $300 \mathrm{~K}$ to $200 \mathrm{~K}$ leads to a Schottky barrier height $\Phi_{\mathrm{B}}$ of $270 \mathrm{meV}$ at $V_{\mathrm{g}}=0 \mathrm{~V}$. Moreover, both for hole and electron transport regions, the $I_{\text {on }} / I_{\text {off }}$ do not improve too much when the temperature is below $200 \mathrm{~K}$. This could be due to the presence of tunneling through defect states inside the $\mathrm{MgO}$ tunnel barrier. ${ }^{32}$ This phenomenon has also been observed in both carbon nanotube $^{33}$ and graphene nanoribbon transistors. ${ }^{34}$

\section{E. Determination of Schottky barrier height}

In order to extract the Schottky barrier height $\left(\Phi_{\mathrm{B}}\right)$ of $\mathrm{Au} / \mathrm{Co} / \mathrm{MgO}$ contact on BP [inset of Fig. 3(a)] and understand the effect of $V_{\mathrm{g}}$ on $\Phi_{\mathrm{B}}$, we have measured the $I_{\mathrm{ds}}-V_{\mathrm{ds}}$ characteristics with different $V_{\mathrm{g}}$ from $140 \mathrm{~K}$ to $200 \mathrm{~K}$ [Fig. 3(a)]. In this temperature range, the thermionic emission transport mechanism through the Schottky barrier can be mainly considered. We have employed a two-dimensional thermionic emission equation describing the electrical transport through the Schottky barrier into the BP channel, ${ }^{35}$

$$
I_{\mathrm{ds}}=A A^{*} T^{1.5} \exp \left[-\frac{q}{k_{\mathrm{B}} T}\left(\Phi_{\mathrm{B}}-\frac{V_{\mathrm{ds}}}{n_{0}}\right)\right],
$$

where $A$ is the contact area, $A^{*}$ is the Richardson constant, $q$ is the hole charge, and $n_{0}$ is the ideality factor. Figure 3(b) shows the Arrhenius plot $\left[\ln \left(I_{\mathrm{ds}} / T^{-3 / 2}\right)\right.$ vs. $\left.1000 / T\right]$ for different $V_{\mathrm{ds}}$. The slopes $S\left(V_{\mathrm{ds}}\right)$ extracted from the Arrhenius plot follow a linear dependence with $V_{\mathrm{ds}}: S\left(V_{\mathrm{ds}}\right)=-\left(q / 1000 k_{\mathrm{B}}\right)\left(\Phi_{\mathrm{B}}-V_{\mathrm{ds}} / n_{0}\right)$, as displayed in Fig. 3(c). Then the Schottky barrier height can be evaluated from the extrapolated value at zero $V_{\mathrm{ds}}\left[S_{0}\right.$ $\left.=-\left(q \Phi_{\mathrm{B}} / 1000 k_{\mathrm{B}}\right)\right]$. A similar procedure has been used to determine $\Phi_{\mathrm{B}}$ with different $V_{\mathrm{g}}$, as shown in Fig. 3(d).

As shown in the inset of Fig. 3(d), the back-gate mainly plays two roles. One is to modulate the Fermi level inside the BP bandgap yielding a change of the carrier density in the channel. The second role is to modify the Schottky barrier profile and depletion layer width. In Fig. 3(d), we can identify two regions from the variation of $\Phi_{\mathrm{B}} v s$. $V_{\mathrm{g}}$. For $V_{\mathrm{g}}>-15 \mathrm{~V}$ when the depletion layer is thick, the thermionic emission dominates, and this results in a large linear increase of $\Phi_{\mathrm{B}}$ at low $\left|V_{\mathrm{g}}\right|$. Note here, the $\Phi_{\mathrm{B}}$ obtained at $V_{\mathrm{g}}=0 \mathrm{~V}$ is $\sim 200 \mathrm{meV}$, which is in good agreement with the value of $270 \mathrm{meV}$ estimated from $I_{\mathrm{on}} / I_{\mathrm{off}}$ ratio at $V_{\mathrm{g}}=0 \mathrm{~V}$. For $V_{\mathrm{g}}<-15 \mathrm{~V}$, the tunneling current through the thin Schottky barrier impinges on the linearity of $\Phi_{\mathrm{B}}$. The real value of $\Phi_{\mathrm{B}}$ for $\mathrm{Co} / \mathrm{MgO}$ on $\mathrm{BP}$ is obtained at the point of the onset of the deviation $\left(V_{\mathrm{f}}=-15 \mathrm{~V}\right)$ equaling thus $37 \mathrm{meV}$, which is corresponding to the flat band condition. This $\Phi_{\mathrm{B}}$ value is much smaller than the recent reports of Py contacts $(110-200 \mathrm{meV}),{ }^{36}$ $\mathrm{Au} / \mathrm{Ti} / \mathrm{BP}$ contacts $(200 \mathrm{meV}),{ }^{18}$ and $\mathrm{Co} / \mathrm{TiO}_{2} /$ contacts
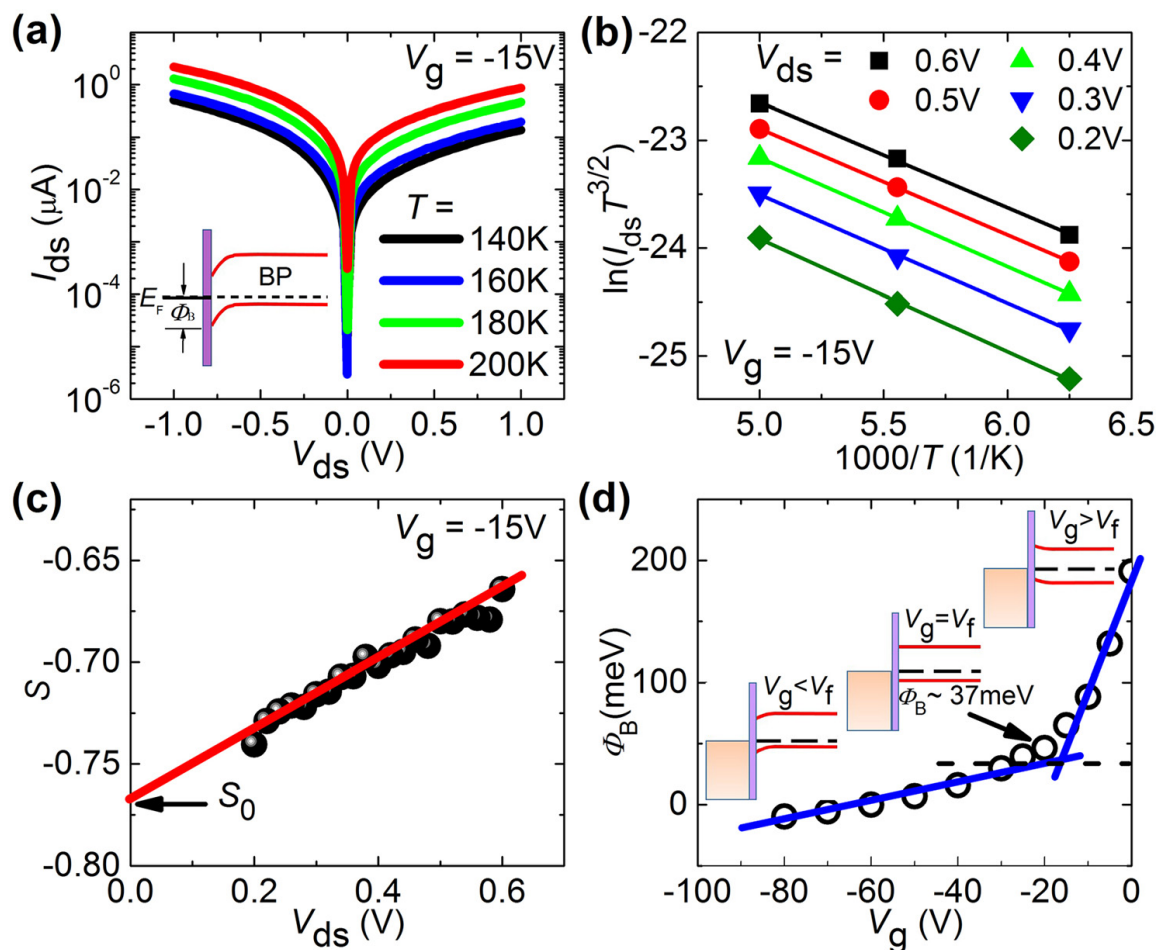

(d)

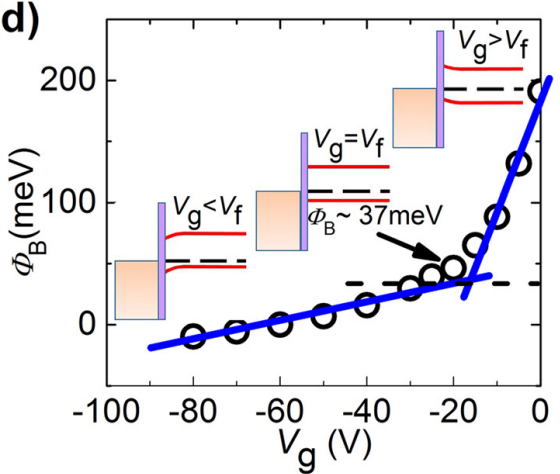

FIG. 3. (a) $I_{\mathrm{ds}}-V_{\mathrm{ds}}$ characteristics for temperatures between 140 and $200 \mathrm{~K}$ under $V_{\mathrm{g}}=-15 \mathrm{~V}$. Inset: Schematics of the Schottky barrier height for $\mathrm{Co} /$ $\mathrm{MgO}$ contact on $p$-type BP. (b) $\ln \left(I_{\mathrm{ds}} /\right.$ $\left.T^{3 / 2}\right)$ versus $1000 / T$ at different drainsource bias $\left(V_{\mathrm{ds}}\right)$, in an Arrhenius plot with linear fits in the temperature range from $160 \mathrm{~K}$ to $200 \mathrm{~K}$. (c) Bias dependence of the slope $(S)$ from linear fits. The slope at zero $V_{\mathrm{ds}}\left(S_{0}\right)$ is used to extract the Schottky barrier height $\Phi_{\mathrm{B}}$. (d) Schottky barrier height $\Phi_{\mathrm{B}}$ extracted at different back-gate voltages. The real $\Phi_{\mathrm{B}}(37 \mathrm{meV})$ for $\mathrm{Au} /$ $\mathrm{Co} / \mathrm{MgO}$ on $\mathrm{BP}$ is obtained with the flat band condition $\left(V_{\mathrm{g}}=V_{\mathrm{f}}=-15 \mathrm{~V}\right)$, which is defined at the cross point from the linear response of $\Phi_{\mathrm{B}}$ with $V_{\mathrm{g}}$ (blue solid lines). Insert: band diagram of $\mathrm{Co} / \mathrm{MgO} / \mathrm{BP}$ interfaces with different back-gate voltages. 

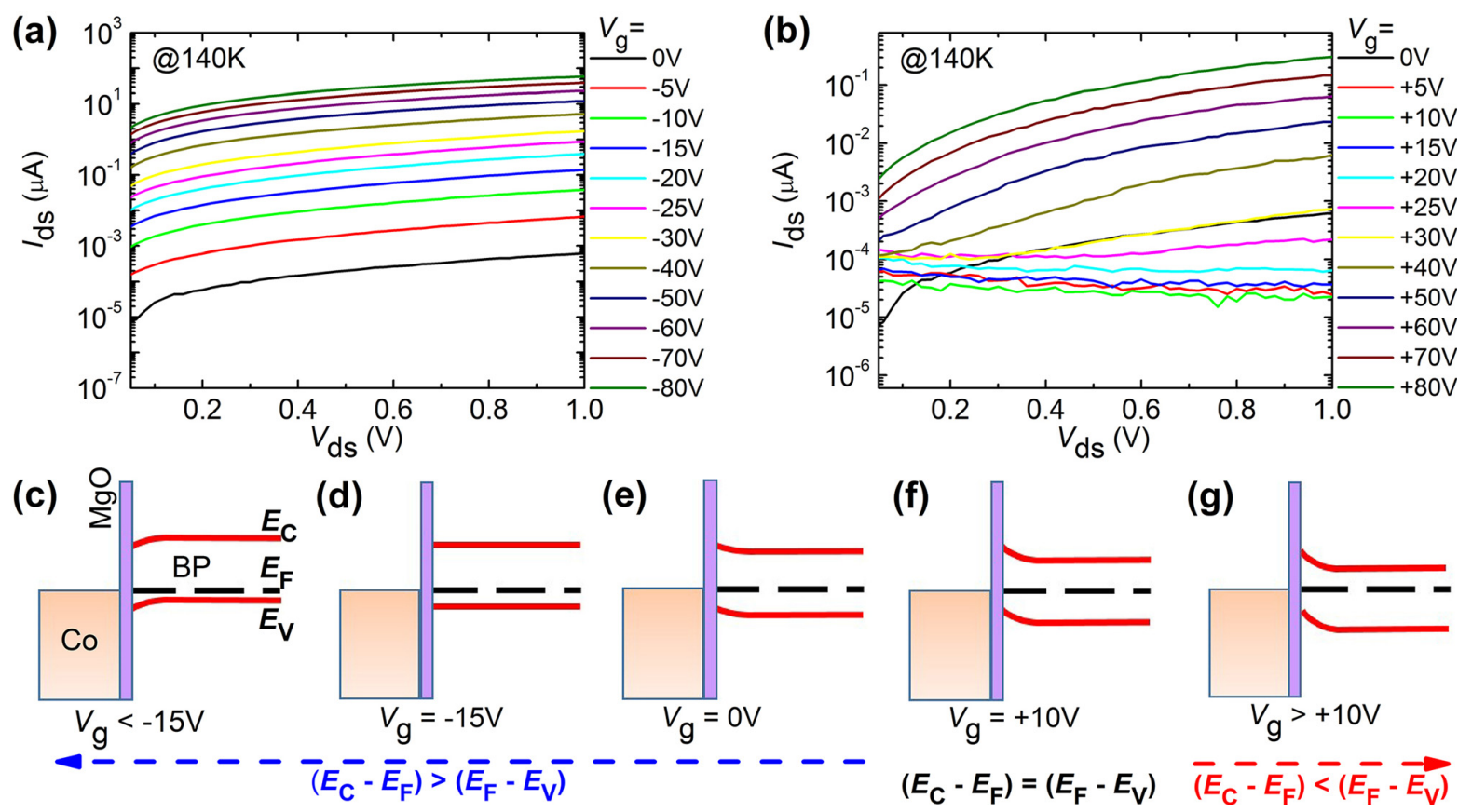

FIG. 4. Characteristics of $I_{\mathrm{ds}}-V_{\mathrm{ds}}$ at $140 \mathrm{~K}$, measured under (a) different negative back-gate voltages and (b) different positive back-gate voltages. (c)-(g) Band structure of $\mathrm{Co} / \mathrm{MgO} / \mathrm{BP}$ interface modulated under different back-gate voltages. (c) $V_{\mathrm{g}}<-15 \mathrm{~V}$; (d) $V_{\mathrm{g}}=-15 \mathrm{~V}$; (e) $V_{\mathrm{g}}=0 \mathrm{~V}$; (f) $V_{\mathrm{g}}=+10 \mathrm{~V}$; (g) $V_{\mathrm{g}}>+10 \mathrm{~V}$.

$(50 \mathrm{meV}) .^{24}$ The efficient lowing $\Phi_{\mathrm{B}}$ by inserting thin tunnel barrier between the ferromagnetic metal and 2D materials have also been reported in TMDs systems. ${ }^{25,37,38}$ This result of low Schottky barrier by using $\mathrm{Au} / \mathrm{Co} / \mathrm{MgO}$ tunneling contact is important for future application of BP based FET and spintronics.

\section{F. Modulation of band structure by back-gate voltage}

In the end, to have a global view of the band structure modulation by the back-gate voltage, we have measured $I_{\mathrm{ds}}$ vs. $V_{\mathrm{ds}}$ with applying different $V_{\mathrm{g}}$ at $140 \mathrm{~K}$. Figures 4 (a) and 4(b) show $I_{\mathrm{ds}}-V_{\mathrm{ds}}$ characteristics with negative and positive $V_{\mathrm{g}}$, respectively. From the analysis of $\Phi_{\mathrm{B}} v s$. $V_{\mathrm{g}}$, we have identified that the flat band condition reaches at $V_{\mathrm{g}}=-15 \mathrm{~V}$ [Fig. 3(d)]. In addition, due to the hole transport feature, the band structure can be schematically drawn as in Fig. 4(d) with a situation of $E_{\mathrm{C}}-E_{\mathrm{F}}>E_{\mathrm{F}}-E_{\mathrm{V}}$. For $V_{\mathrm{g}}<-15 \mathrm{~V}$, the depletion layer tilts the band down due to the $E_{\mathrm{F}}$ pinning at the interface [Fig. 4(c)]. With the increase of negative $V_{\mathrm{g}}$, more hole can be injected into BP by crossing the depletion layer. For $V_{\mathrm{g}}>-15 \mathrm{~V}$, the depletion layer tilts the band up at the interface. However, it is not the case that the larger $V_{\mathrm{g}}$ results in larger $I_{\text {ds. }}$. From Fig. 4(b), we can see that when we increase $V_{\mathrm{g}}$ from 0 to $+80 \mathrm{~V}$, the $I_{\mathrm{ds}}$ firstly decreases to a minimum at $V_{\mathrm{g}}=+10 \mathrm{~V}$ and then increases with $V_{\mathrm{g}}$. This indicates that it is hard to inject both holes and electrons at $V_{\mathrm{g}}=+10 \mathrm{~V}$, which should correspond to the condition that $E_{\mathrm{F}}$ is located in the middle of band $\left(E_{\mathrm{C}}-E_{\mathrm{F}}=E_{\mathrm{F}}-E_{\mathrm{V}}\right)$, as shown in Fig. 4(f). When continuing with the increasing $V_{\mathrm{g}}$, both the $E_{\mathrm{F}}$ moving closer to $E_{\mathrm{C}}$ and the width of depletion region becoming thinner make the conditions favorable for the electron injection into the BP conduction band [Fig. 4(g)]. Finally, at zero back-gate voltage, the $\mathrm{BP}$ shows a hole transport behavior $\left(E_{\mathrm{C}}-E_{\mathrm{F}}>E_{\mathrm{F}}-E_{\mathrm{V}}\right)$, while the depletion layer tilts up [Fig. 4(e)]. This could be related to the defects in the contact regions, which effectively pin the Fermi level at $\mathrm{MgO} / \mathrm{BP}$ interface.

\section{CONCLUSION}

In summary, we have studied the electrical transport properties in multilayer BP based FET device with $\mathrm{Au} / \mathrm{Co} /$ $\mathrm{MgO}$ tunneling contacts. In the hole dominated transport region, we have measured a minimum of Schottky barrier height of $37 \mathrm{meV}$ for $\mathrm{Au} / \mathrm{Co} / \mathrm{MgO}$ contact on $\mathrm{BP}$. Moreover, the transistor $\mathrm{ON} / \mathrm{OFF}\left(I_{\mathrm{on}} / I_{\text {off }}\right)$ ratio is obtained as large as $10^{7}$ at $20 \mathrm{~K}$ and $10^{5}$ at $300 \mathrm{~K}$. A systematic study of the temperature and back-gate voltage dependent conduction measurements has been performed to understand the modulation of the band structure and the ambipolar behavior. This demonstration of high ON/OFF ratio and low Schottky barrier height by using $\mathrm{Au} / \mathrm{Co} / \mathrm{MgO}$ tunneling contacts reveal a great potential promising for spintronics applications with multilayer black phosphorus field effect transistor.

\section{ACKNOWLEDGMENTS}

This work was supported by the French National Research Agency (ANR) MoS2ValleyControl project (Grant No. ANR-14-CE26-0017-04) and the joint ANR-National Natural Science Foundation of China (NNSFC) ENSEMBLE project (Grant Nos. ANR-14-CE26-0028-01 and NNSFC 61411136001). This work was also supported partly by the French PIA project "Lorraine Université d'Excellence", reference No. ANR-15-IDEX-04-LUE. The experiments were 
performed using the equipment from the platform TUBE-Davm funded by FEDER (EU), ANR, the Region Lorraine, and Grand Nancy.

${ }^{1}$ S. Novoselov, A. K. Geim, S. V. Morozov, D. Jiang, Y. Zhang, S. V. Dubonos, I. V. Grigorieva, and A. A. Firsov, "Electric field effect in atomically thin carbon films," Science 306, 666 (2004).

${ }^{2}$ K. A. H. Castro Neto, F. Guinea, N. M. R. Peres, K. S. Novoselov, and A. K. Geim, "The electronic properties of graphene," Rev. Mod. Phys. 81, 109 (2009).

${ }^{3}$ G. Fiori, F. Bonaccorso, G. Iannaccone, T. Palacios, D. Neumaier, A Seabaugh, S. K. Banerjee, and L. Colombo, "Electronics based on twodimensional materials," Nat. Nanotechnol. 9, 768 (2014).

${ }^{4}$ K. F. Mak, C. Lee, J. Hone, J. Shan, and T. F. Heinz, "Atomically thin $\mathrm{MoS}_{2}$ a new direct-gap semiconductor," Phys. Rev. Lett. 105, 136805 (2010)

${ }^{5}$ M. Xu, T. Liang, M. Shi, and H. Chen, "Graphene-like two-dimensional materials," Chem. Rev. 113, 3766 (2013).

${ }^{6}$ D. Jariwala, V. K. Sangwan, L. J. Lauhon, T. J. Marks, and M. C. Hersam, "Emerging device applications for semiconducting two-dimensional transition metal dichalcogenides," ACS Nano 8, 1102 (2014).

B. Radisavljevic, A. Radenovic, J. Brivio, V. Giacometti, and A. Kis, "Single-layer $\mathrm{MoS}_{2}$ transistors," Nat. Nanotechnol. 6, 147 (2011).

${ }^{8}$ D. Xiao, G. Liu, W. Feng, X. Xu, and W. Yao, "Coupled spin and valley physics in monolayers of $\mathrm{MoS}_{2}$ and other group-VI dichalcogenides," Phys. Rev. Lett. 108, 196802 (2012).

${ }^{9}$ S. Liang, H. Yang, P. Renucci, B. Tao, P. Laczkowski, S. Mc-Murtry, G. Wang, X. Marie, J. George, S. Petit-Watelot, A. Djeffal, S. Mangin, H. Jaffres, and Y. Lu, "Electrical spin injection and detection in molybdenum disulfide multilayer channel," Nat. Commun. 8, 14947 (2017).

${ }^{10}$ D. Pacilé, J. C. Meyer, Ç. Ö. Girit, and A. Zettl, "The two-dimensiona phase of boron nitride: Few-atomic-layer sheets and suspended membranes," Appl. Phys. Lett. 92, 133107 (2008).

${ }^{11}$ Q. H. Wang, K. Kalantar-Zadeh, A. Kis, J. N. Coleman, and M. S. Strano, "Electronics and optoelectronics of two- dimensional transition metal dichalcogenides," Nat. Nanotechnol. 7, 699 (2012).

${ }^{12}$ K. Lee, R. Gatensby, N. McEvoy, T. Hallam, and G. S. Duesberg, "High performance sensors based on molybdenum disulfide thin films," Adv. Mater. 25, 6699 (2013).

${ }^{13}$ M. Bernardi, M. Palummo, and J. C. Grossman, "Extraordinary sunlight absorption and one nanometer thick photovoltaics using two-dimensional monolayer materials," Nano Lett. 13, 3664 (2013).

${ }^{14}$ R. Kurapati, K. Kostarelos, M. Prato, and A. Bianco, "Biomedical uses for 2D materials beyond graphene: Current advances and challenges ahead," Adv. Mater. 28, 6052 (2016).

${ }^{15}$ X. Zhang, L. Hou, A. Ciesielski, and P. Samorì, "2D materials beyond graphene for high-performance energy storage applications," Adv. Energy Mater. 6, 1600671 (2016)

${ }^{16} \mathrm{~V}$. Tran, R. Soklaski, Y. Liang, and L. Yang, "Layer-controlled band gap and anisotropic excitons in few-layer black phosphorus," Phys. Rev. B 89, 235319 (2014)

${ }^{17}$ H. Liu, A. T. Neal, Z. Zhu, Z. Luo, X. Xu, D. Tománek, and P. D. Ye, "Phosphorene: An unexplored 2D semiconductor with a high hole mobility," ACS Nano 8, 4033 (2014).

${ }^{18}$ L. Li, Y. Yu, G. Ye, Q. Ge, X. Ou, H. Wu, D. Feng, X. Chen, and Y. Zhang, "Black phosphorus field-effect transistors," Nat. Nanotechnol. 9 372 (2014).
${ }^{19}$ S. P. Koenig, R. A. Doganov, H. Schmidt, A. H. Castro Neto, and B. Özyilmaz, "Electric field effect in ultrathin black phosphorus," Appl. Phys. Lett. 104, 103106 (2014)

${ }^{20} \mathrm{~F}$. Xia, H. Wang, and Y. Jia, "Rediscovering black phosphorus as an anisotropic layered material for optoelectronics and electronics," Nat. Commun. 5, 4458 (2014).

${ }^{21}$ M. Buscema, D. J. Groenendijk, S. I. Blanter, G. A. Steele, H. S. J. van der Zant, and A. Castellanos-Gomez, "Fast and broadband photoresponse of few-layer black phosphorus field-effect transistors," Nano Lett. 14, 3347 (2014).

${ }^{22}$ A. Avsar, J. Y. Tan, M. Kurpas, M. Gmitra, K. Watanabe, T. Taniguchi, J. Fabian, and B. Özyilmaz, "Gate-tunable black phosphorus spin valve with nanosecond spin lifetimes," Nat. Phys. 13, 888 (2017).

${ }^{23}$ Y. Du, H. Liu, Y. Deng, and P. D. Ye, "Device perspective for black phosphorus field-effect transistors: Contact resistance, ambipolar and scaling," ACS Nano 8, 10035 (2014).

${ }^{24}$ M. V. Kamalakar, B. N. Madhushankar, A. Dankert, and S. P. Dash, "Low Schottky barrier black phosphorus field-effect devices with ferromagnetic tunnel contacts," Small 11, 2209 (2015).

${ }^{25}$ J. Chen, P. M. Odenthal, A. G. Swartz, G. C. Floyd, H. Wen, K. Y. Luo, and R. K. Kawakami, "Control of Schottky barriers in single layer $\mathrm{MoS}_{2}$ transistors with ferromagnetic contacts," Nano Lett. 13, 3106 (2013).

${ }^{26}$ S. M. Sze and K. K. Ng, Physics of Semiconductor Devices (Wiley, 2007).

${ }^{27}$ K. Kaasbjerg, K. S. Thygesen, and K. W. Jacobsen, "Phonon limited mobility in n-type single-layer $\mathrm{MoS}_{2}$ from first principles," Phys. Rev. B 85, 115317 (2012).

${ }^{28}$ E. H. Hwang and S. Das Sarma, "Acoustic phonon scattering limited carrier mobility in two-dimensional extrinsic graphene," Phys. Rev. B 77, 115449 (2008)

${ }^{29}$ E. Mariani, A. J. Pearce, and F. von Oppen, "Fictitious gauge fields in bilayer graphene," Phys. Rev. B 86, 165448 (2012).

${ }^{30}$ H. Ochoa, E. V. Castro, M. I. Katsnelson, and F. Guinea, "Temperaturedependent resistivity in bilayer graphene due to flexural phonons," Phys. Rev. B 83, 235416 (2011)

${ }^{31}$ F. Xia, D. B. Farmer, Y. Lin, and P. Avouris, "Graphene field-effect transistors with high on/off current ratio and large transport band gap at room temperature," Nano Lett. 10, 715 (2010).

${ }^{32}$ Y. Lu, M. Tran, H. Jaffrès, P. Seneor, C. Deranlot, F. Petroff, J.-M. George, B. Lépine, S. Ababou, and G. Jézéquel, "Spin-polarized inelastic tunneling through insulating barriers," Phys. Rev. Lett. 102, 176801 (2009).

${ }^{33}$ J. Appenzeller, M. Radosavljevic, J. Knoch, and P. Avouris, "Tunneling versus thermionic emission in one-dimensional semiconductors," Phys. Rev. Lett. 92, 048301 (2004)

${ }^{34}$ Z. Chen, Y. M. Lin, M. J. Rooks, and P. Avouris, "Graphene nano-ribbon electronics," Physica E 40, 228 (2007).

${ }^{35}$ A. Anwar, B. Nabet, J. Culp, and F. Castro, "Effects of electron confinement on thermionic emission current in a modulation doped heterostructure," J. Appl. Phys. 85, 2663 (1999).

${ }^{36}$ Y. Anugrah, M. C. Robbins, P. A. Crowell, and S. J. Koester, "Determination of the Schottky barrier height of ferromagnetic contacts to few-layer phosphorene," Appl. Phys. Lett. 106, 103108 (2015).

${ }^{37}$ W. Wang, Y. Liu, L. Tang, Y. Jin, T. Zhao, and F. Xiu, "Controllable Schottky barriers between $\mathrm{MoS}_{2}$ and permalloy," Sci. Rep. 4, 6928 (2014).

${ }^{38}$ A. Dankert, L. Langouche, M. V. Kamalakar, and S. P. Dash, "High-performance molybdenum disulfide field-effect transistors with spin tunnel contacts," ACS Nano 8, 476-482 (2014). 